Supporting Information for

\title{
Controlled Synthesis of Pure-Phase GaAs Nanowires through Shear Tension
}

Yubin Kang, ${ }^{1, \#}$ Guangren Na, ${ }^{2, \#}$ Dengkui Wang, ${ }^{1}$ Jilong Tang, ${ }^{1}$ Lijun Zhang, ${ }^{2}$ Yabing Shan, ${ }^{3}$ Chunxiao Cong, ${ }^{3}$ Zhipeng Wei, ${ }^{1, *}$ and Rui Chen ${ }^{4, *}$

${ }^{1}$ State Key Laboratory of High Powder Semiconductor Lasers, Changchun University of Science and Technology, Changchun 130022, China.

${ }^{2}$ State Key Laboratory of Integrated Optoelectronics, Key Laboratory of Automobile Materials of MOE, School of Materials Science and Engineering, Jilin University, Changchun 130012, China.

${ }^{3}$ State Key Laboratory of ASIC and System, School of Information Science and Technology, Fudan University, Shanghai 200433, China.

${ }^{4}$ Department of Electrical and Electronic Engineering, Southern University of Science and Technology, Guangdong 518055, China.

\footnotetext{
\# Y. Kang and G. Na contributed equally to this work

* E-mail: zpweicust@126.com and chenr@sustech.edu.cn
} 


\section{EXPERIMENTAL METHODS}

Substrate Treatment. The $n$-type Si (111) substrates were treated to remove of partly native $\mathrm{SiO}_{2}$ layer by using 1:10 buffered hydrofluoric acid solution (BHF). Then, they were ultrasonically cleaned with ethanol and deionized (DI) water for $5 \mathrm{~min}$, respectively. Subsequently, the substrates were dried by nitrogen. Then, the substrates were treated by ultra-high vacuum environment of our MBE system (DCA P600). First, a halogen lamp degasses the substrates holder at $\sim 200{ }^{\circ} \mathrm{C}$ for $2 \mathrm{~h}$ in the MBE load-lock system (between $10^{-8}$ and $10^{-9}$ Torr). Second, the substrates were heated to $\sim 400{ }^{\circ} \mathrm{C}$ in a separate chamber for $2 \mathrm{~h}$ at a pressure $\sim 10^{-10}$ Torr. A third and last degassing were performed in the growth chamber directly prior to growth at $750{ }^{\circ} \mathrm{C}$ for $30 \mathrm{~min}$.

Nanowire Growth. The self-catalyzed GaAs and GaAs/GaAsSb NWs were grown on n-type Si (111) substrates by solid-source molecular beam epitaxy (MBE). Ga droplets were deposited on Si substrates at beam equivalent pressure of $6.2 \times 10^{-8}$ Torr and the deposition time of $28 \mathrm{~s}$. Subsequently, the GaAs core was grown with a Ga beam equivalent pressure, V/III ratio and substrate temperature of $6.2 \times 10^{-8}$ Torr, 25.8, and $620{ }^{\circ} \mathrm{C}$ for $10 \mathrm{~min}$. Then, the GaAsSb shell was grown with a Ga beam equivalent pressure, $\mathrm{V} / \mathrm{III}$ ratio, $\mathrm{Sb} / \mathrm{Ga}$ ratio, $\mathrm{Sb} /(\mathrm{As}+\mathrm{Sb})$ ratio, substrate temperature of $6.2 \times 10^{-8}$ Torr, $27.58 \sim 30.97,1.80 \sim 5.00,6.43 \sim 16.67$, and $\sim 620{ }^{\circ} \mathrm{C}$ for $10 \mathrm{~min}$, respectively. The GaAs NWs was grown under the same conditions as the GaAs core for $20 \mathrm{~min}$. After the growth, the Ga and As were switched off and the samples were 
cooled under $\mathrm{Sb}$ overpressure to $300{ }^{\circ} \mathrm{C}$. Finally, the samples were cooled to room temperature naturally.

Material Characterization. The morphology of GaAs/GaAsSb NWs were examined by scanning electron microscopy (SEM, HITACHI S-4800). The microstructure and composition of GaAs/GaAsSb core-shell NWs were determined by a transmission electron microscopy (TEM, FEI Talos F200S) with associated EDXs and a double aberration-corrected JEOL ARM200F (scanning) transmission electron microscope (TEM/STEM). Micro-Raman spectra were collected from a spectrometer (Alpha 300R WiTec) equipped with a $\times 100$ microscope objective and a piezo stage, which enables to scan along the NW with a precision of $10 \mathrm{~nm}$. All $\mu$-Raman spectra were obtained in a backscattering configuration. A $532 \mathrm{~nm}$ laser was used for the excitation of the individual GaAs and GaAs/GaAsSb core-shell NWs. The power and the spot diameter of the incident laser were about $3 \mathrm{~mW}$ and $300 \mathrm{~nm}$, respectively. The polarization direction of the incident laser was perpendicular to the axis. The Raman spectral results of large-area NWs were conducted with a Raman Spectrometer (Raman, LabRAM HR Evolution, HORIBA Scientific, Japan) with a laser excitation wavelength of $532 \mathrm{~nm}$ and the spot diameter is $400 \mu \mathrm{m}$.

Detector Fabrication. For the fabrication of GaAs/GaAsSb NW APD, the NWs were transferred onto a p-type $\mathrm{Si}$ wafer with $300 \mathrm{~nm} \mathrm{SiO}$. The $\mathrm{Cr} / \mathrm{Au}(20 / 80 \mathrm{~nm})$ electrodes on both ends of GaAs/GaAsSb NW was prepared by electron beam 
lithography, metal evaporation and lift-off processes. Before evaporation, the GaAsSb shell on the core-shell segment was removed by HF etching.

Device Characterization. The optoelectronic properties of individual GaAs/GaAsSb APD were characterized by semiconductor device parameter analyzer (Agilent 4155C). For photodetection performance measurement, the light wavelength and the light intensity are $532 \mathrm{~nm}$ and $77.36 \mathrm{~mW} / \mathrm{cm}^{2}$, respectively.

First Principles Calculations. Our first-principles density functional theory calculations were carried out by using the plane-wave projected augmented wave pseudopotential approach as implemented in the Vienna Ab-initio Simulation Package code. ${ }^{1,2}$ The exchange and correlation functional were treated using the generalized gradient approximation of Perdew, Burke, and Ernzerhof (PBE) ${ }^{3,4}$ To simulate the effect of stress of GaAsSb shell, lattice constants obtained from experiment of $\mathrm{ZB}$ and WZ phase GaAs were fixed in the calculations. The kinetic energy cut-off for the plane-wave basis sets was set as $300 \mathrm{eV}$. We used k-point meshes with grid spacing $2 \pi \times 0.08 \AA^{-1}$ or less in electronic Brillouin zone integration. The electronic minimization was performed with a tolerance of $10^{-4} \mathrm{eV}$, and ionic relaxation was performed with a force tolerance of $0.03 \mathrm{eV} \AA^{-1}$ on each ion. Optimized structures of WZ phase and ZB phase were set as initial state and final state for the phase transition process, respectively. 

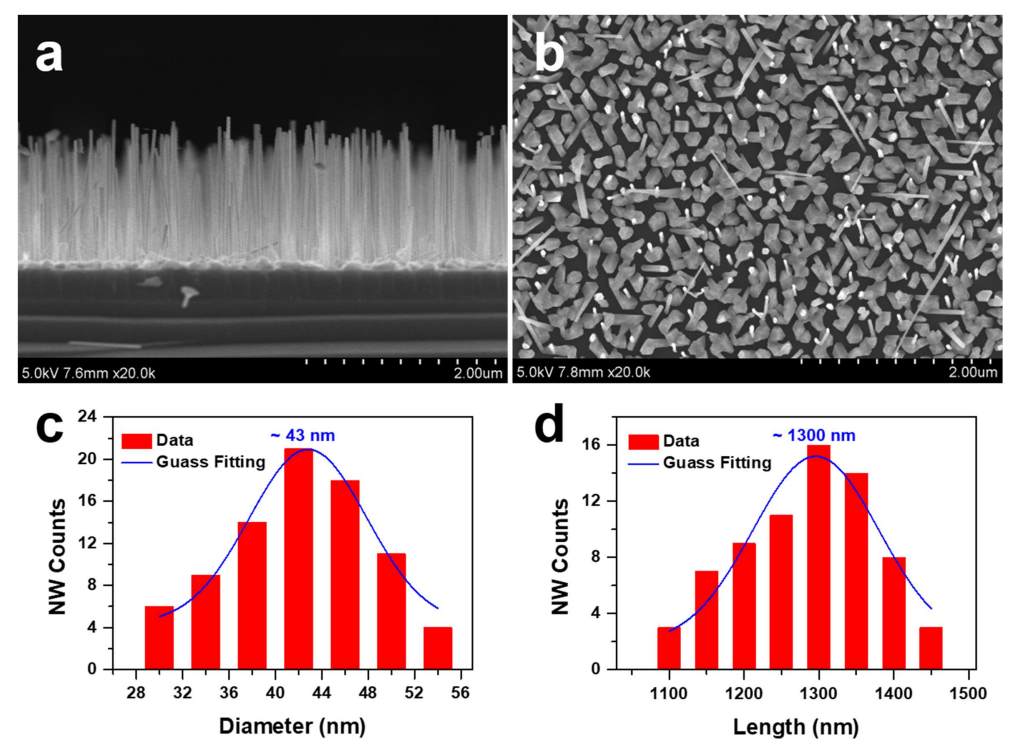

Figure S1. Morphology of the GaAs NWs. $(a, b)$ The side-view and top-view SEM images of GaAs NWs grown on Si (111) substrate. (c) The diameter distribution of GaAs NWs. The average diameter of GaAs NWs is around $43 \mathrm{~nm}$. (d) The length distribution of GaAs NWs. The average length of GaAs NWs is around $1.3 \mu \mathrm{m}$.

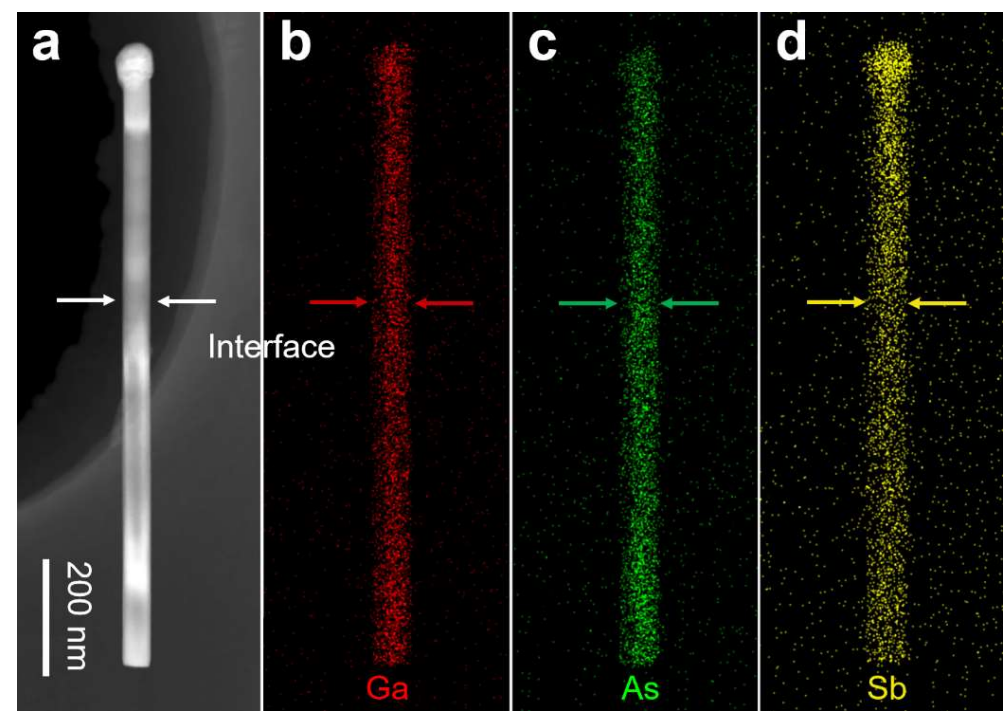

Figure S2. Structure and composition of a typical GaAs/GaAsSb NW. (a) High-resolution high-angle annular dark field (HAADF) scanning transmission electron microscopy (STEM) image of the single GaAs/GaAsSb NW. (b) EDXs mapping of Ga showing a homogeneous composition along the NW. (c,d) EDXs mapping of As, and Sb showing a non-homogeneous composition along the NW. The interface of the GaAs/GaAsSb heterojunction NW is marked by white, red, green, yellow arrows. 

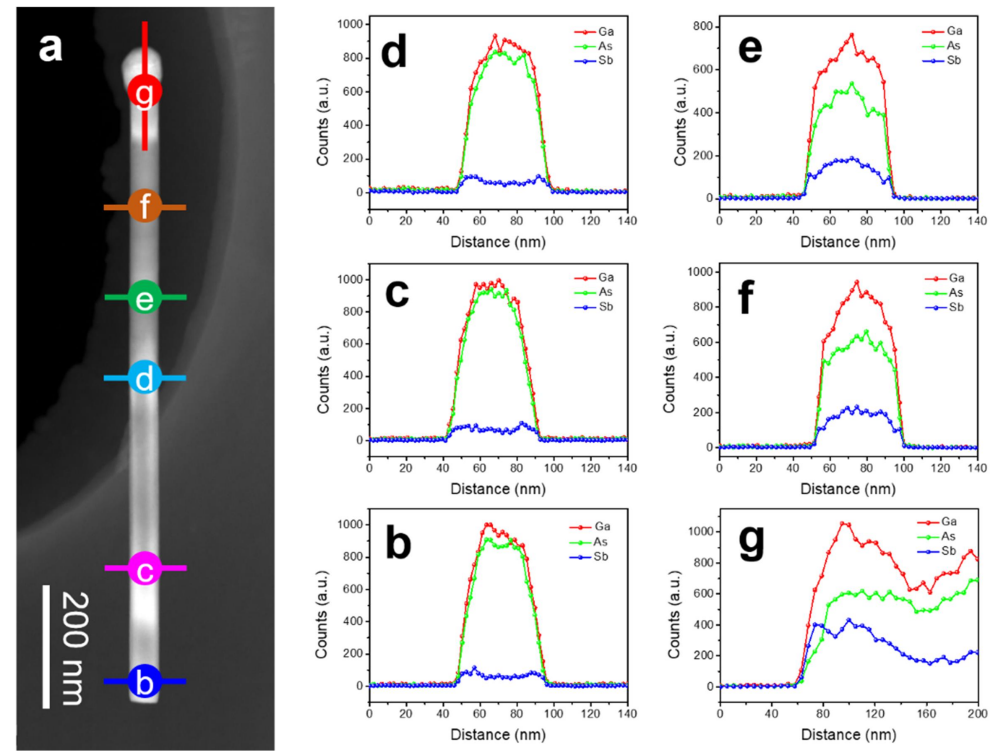

Figure S3. Structure and composition of a typical GaAs/GaAsSb NW. (a) HAADF-STEM micrograph of the single GaAs/GaAsSb NW. (b-g) EDXs line scans along the blue, pink, light blue, green, orange, and red lines marked in (a). The $\mathrm{Sb}$ signal can be clearly identified in the spectra (b-d), indicating the existence of the GaAsSb shell around the GaAs core. The Sb composition is approximately 0.15 0.18 in the GaAsSb shell. The Ga, As and Sb signals can be identified in the spectra (e-g), indicating the existence of the GaAsSb structure without GaAs core.
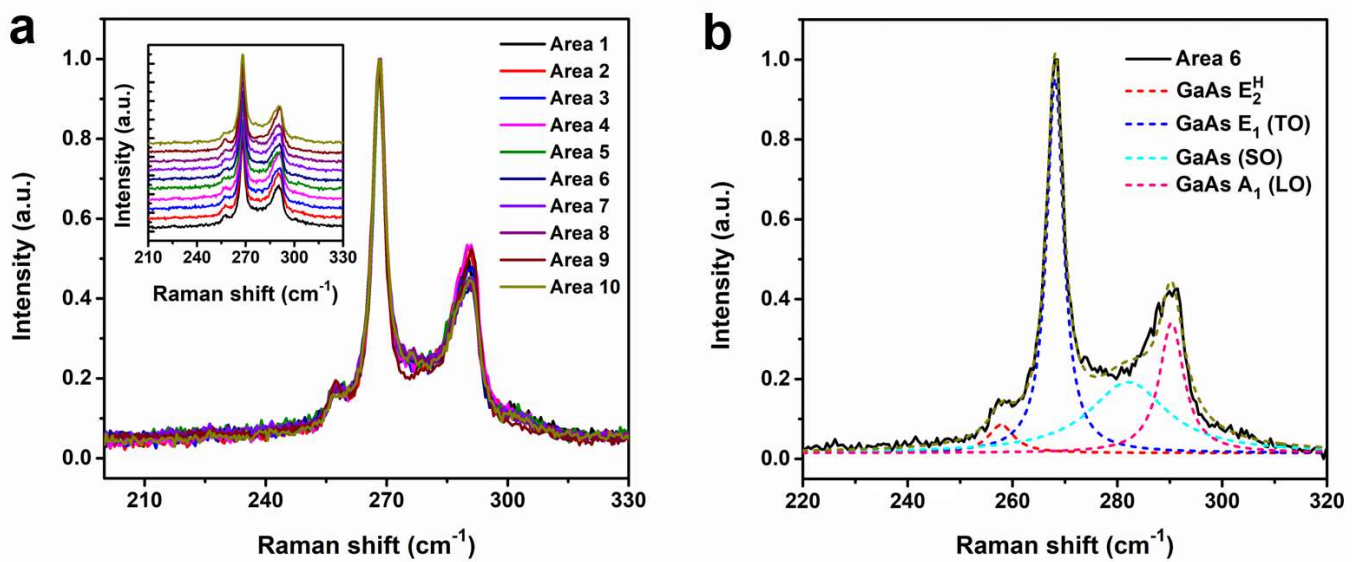

Figure S4. Raman spectra of large-area GaAs NWs (first growth). (a) Raman spectra of GaAs NWs in 10 different regions. (b) Lorentzian line fitting of GaAs NWs (Area $6)$. 

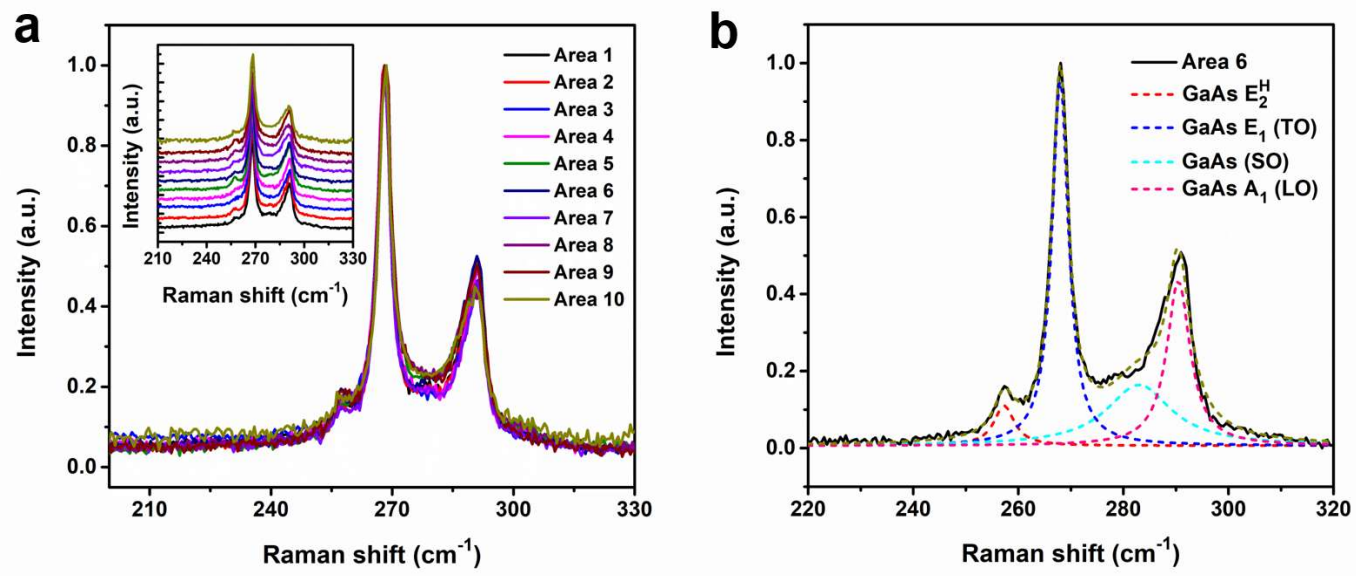

Figure S5. Raman spectra of large-area GaAs NWs (second growth). (a) Raman spectra of GaAs NWs in 10 different regions. (b) Lorentzian line fitting of GaAs NWs (Area 6).

Raman shapes of GaAs NWs obtained twice under the same growth conditions are consistent, as shown in Figure S4 and Figure S5. We see from Figure S4 and Figure S5 that two positions of $257.8 \mathrm{~cm}^{-1}$ and $257.3 \mathrm{~cm}^{-1}$, which are distinguished by the structure characteristic peak of $\mathrm{E}_{2}{ }^{\mathrm{H}}$ mode. Therefore, the structure of GaAs NWs is WZ/ZB mixed-phases.
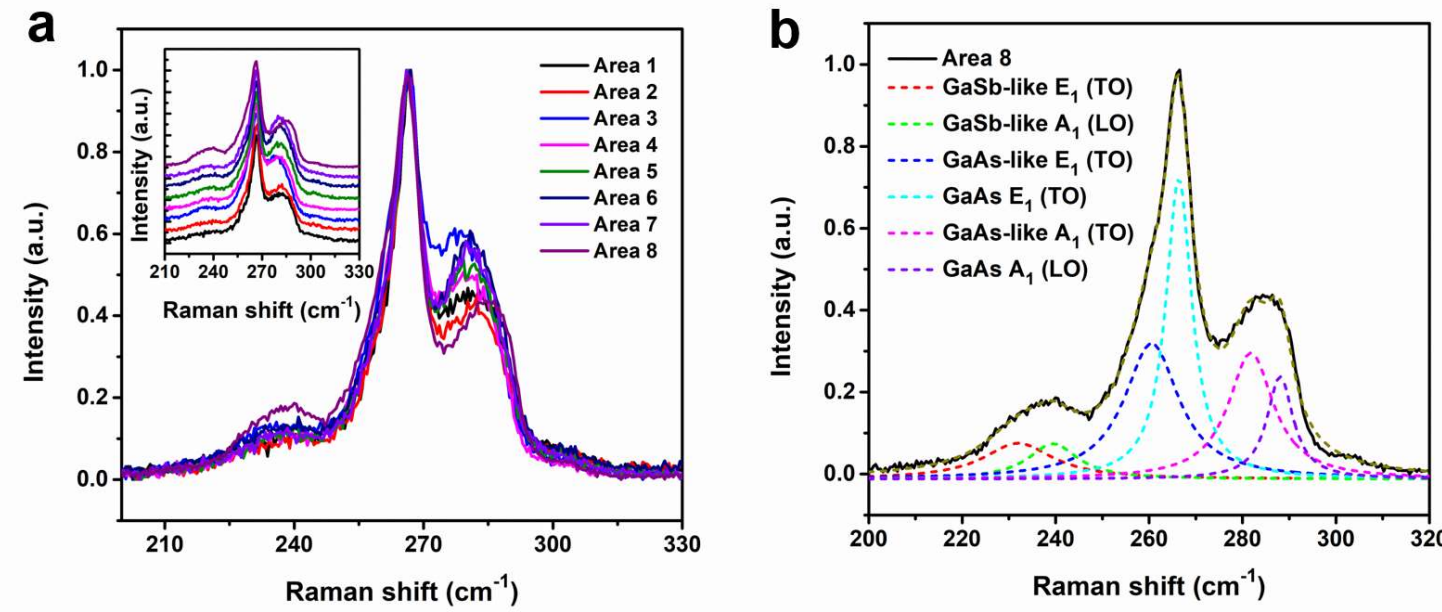

Figure S6. Raman spectra of large-area GaAs/GaAsSb NWs (first growth). (a) Raman spectra of GaAs/GaAsSb NWs in 8 different regions. (b) Lorentzian line fitting of GaAs/GaAsSb NWs (Area 8). 

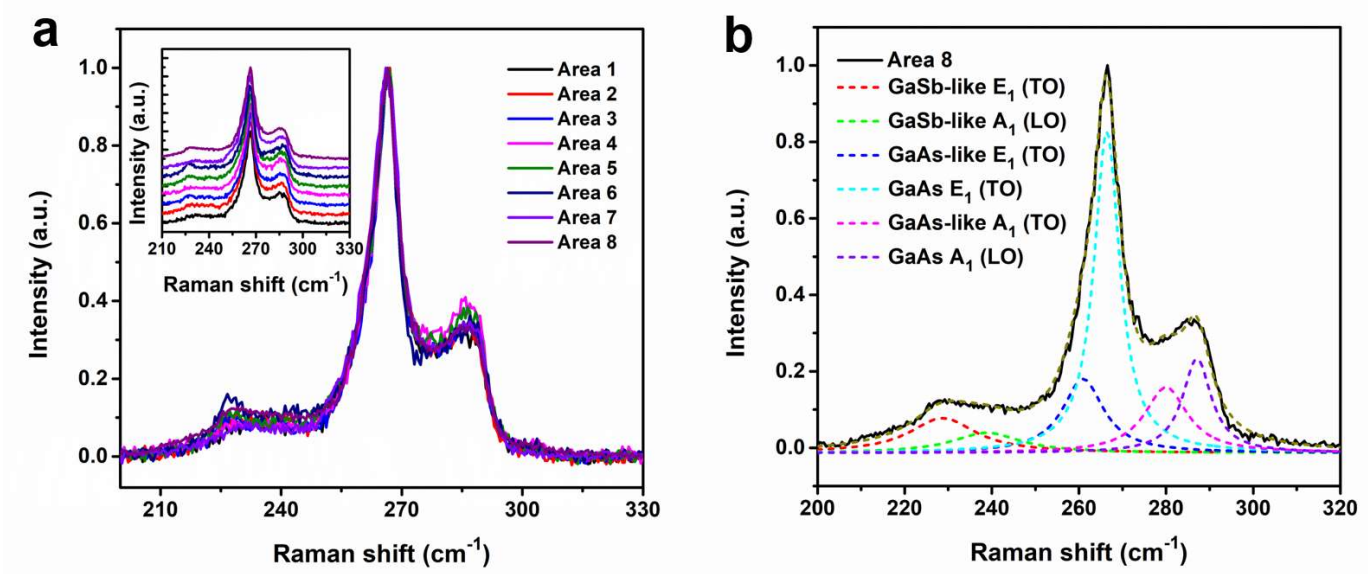

Figure S7. Raman spectra of large-area GaAs/GaAsSb NWs (second growth). (a) Raman spectra of GaAs/GaAsSb NWs in 8 different regions. (b) Lorentzian line fitting of GaAs/GaAsSb NWs (Area 8).

The Raman shapes of GaAs/GaAsSb NWs in different regions obtained twice under the same growth conditions are consistent, as shown in Figure S6 and Figure S7. In addition, GaAs/GaAsSb NWs display the modes of GaSb-like $\mathrm{E}_{1}(\mathrm{TO})$, GaAs-like $\mathrm{E}_{1}(\mathrm{TO}), \mathrm{GaAs} \mathrm{E}_{1}(\mathrm{TO})$ and GaAs-like $\mathrm{A}_{1}(\mathrm{LO})$. The Raman spectral results of large-area GaAs/GaAsSb NWs are not found $\mathrm{E}_{2}{ }^{\mathrm{H}}$ mode.

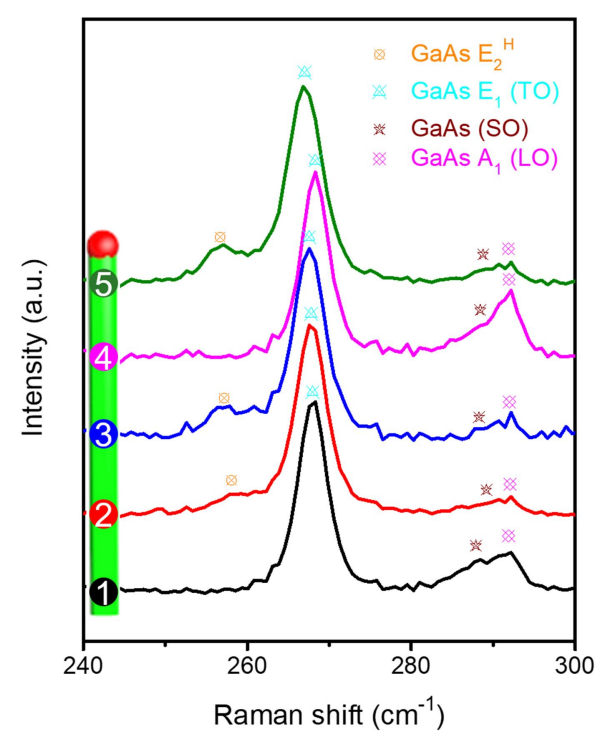

Figure S8. $\mu$-Raman spectra of the different locations along another bare GaAs NW. 
The $\mu$-Raman spectra are performed at various positions along the bare GaAs NW, as shown in Figure S8. The crystal phase of positions 1 and 4 belong to be ZB structure and the other three are $\mathrm{WZ}$ structure, which is distinguished by structural characteristic peak of $\mathrm{E}_{2}{ }^{\mathrm{H}}$ mode. Therefore, the structure of GaAs NW is WZ/ZB mixed-phases.

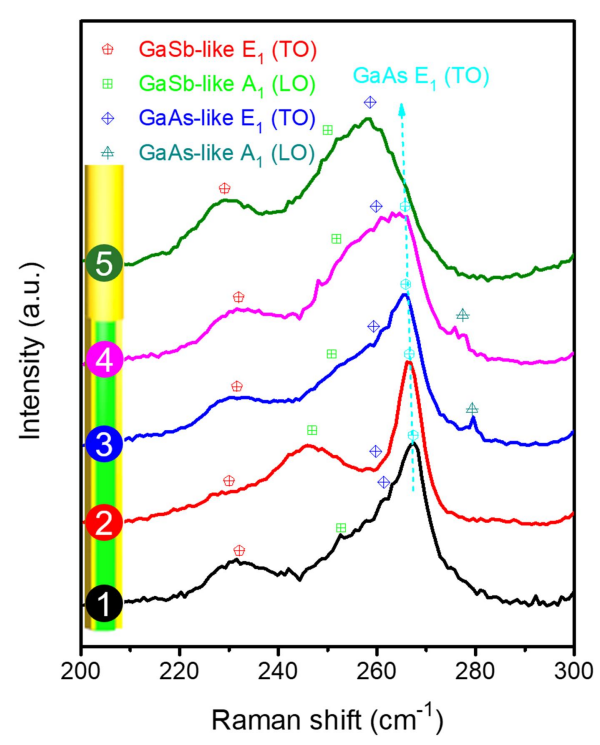

Figure S9. $\mu$-Raman spectra of the different locations along another GaAs/GaAsSb core-shell NW.

The Raman spectra measured along a single GaAs/GaAsSb core-shell NW display the modes of GaSb-like $\mathrm{E}_{1}$ (TO), GaSb-like $\mathrm{A}_{1}$ (LO), GaAs-like $\mathrm{E}_{1}$ (TO), GaAs $E_{1}(\mathrm{TO})$ and GaAs-like $\mathrm{A}_{1}(\mathrm{LO}) . \mathrm{E}_{1}(\mathrm{TO})$ mode of core is marked with a cyan dashed line. In addition, no $\mathrm{E}_{2}{ }^{\mathrm{H}}$ mode can be observed in $\mathrm{GaAs} / \mathrm{GaAsSb}$ core-shell $\mathrm{NW}$, which indicates that the GaAs core is pure ZB phase without any WZ phase. 

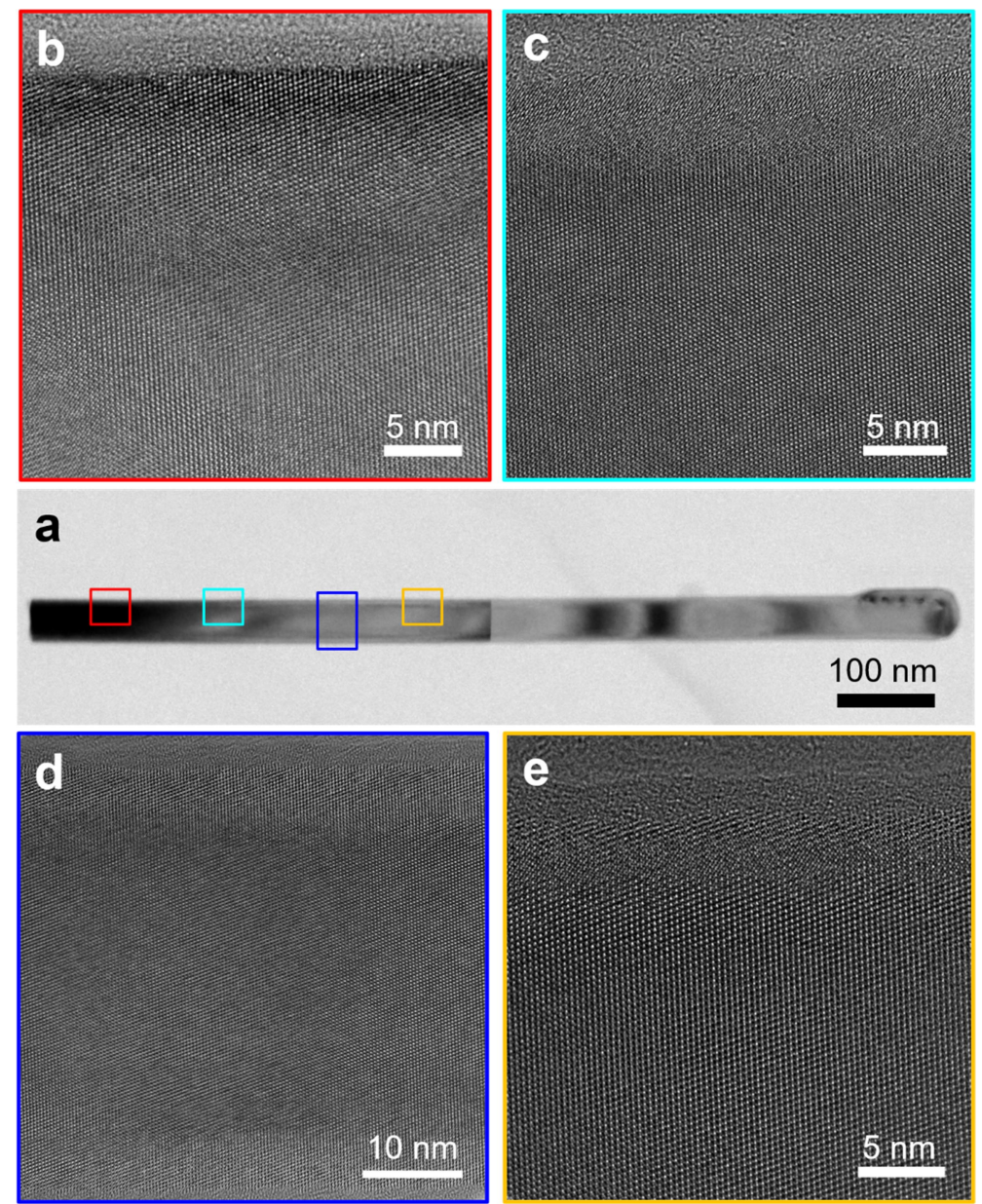

Figure S10. Structure of a typical GaAs/GaAsSb core-shell NW. (a) TEM image showing the morphology of a typical GaAs/GaAsSb core-shell NW. (b-e) HRTEM images taken along [111] zone axis from the bottom to middle regions of the $\mathrm{GaAs} / \mathrm{GaAsSb} \mathrm{NW}$ are shown in (a), respectively.

The HRTEM images show that the GaAs/GaAsSb core-shell NW is pure ZB phase and free of stacking faults. 

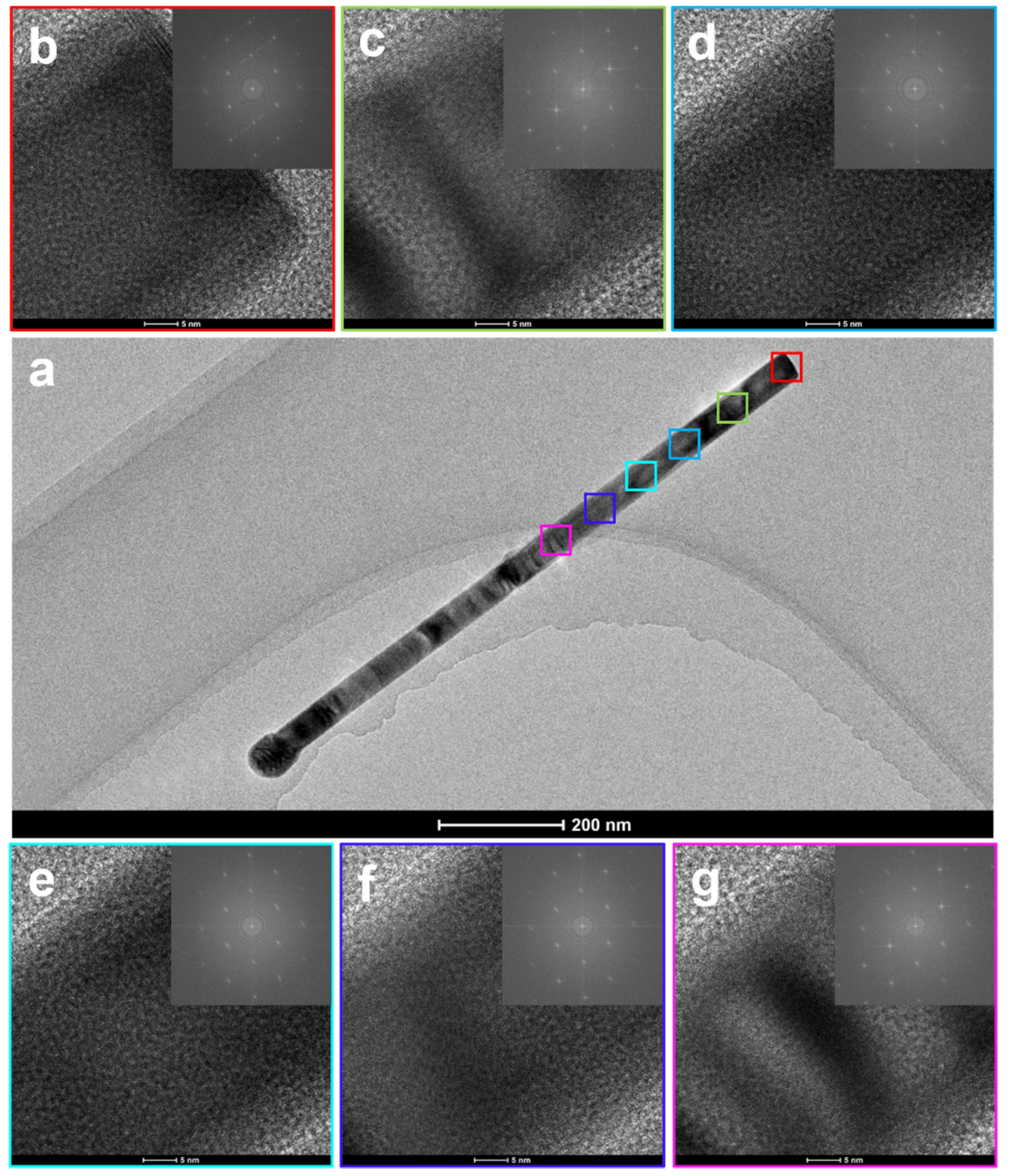

Figure S11. Structure of a typical GaAs/GaAsSb core-shell NW. (a) TEM image of a single GaAs/GaAsSb core-shell NW. (b-g) HRTEM and FFT images taken along [111] zone axis from the bottom to middle regions of the GaAs/GaAsSb NW shown in (a), respectively.

There is a WZ/ZB miscible structure of about 1-2 $\mathrm{nm}$ at the bottom of the NW, as shown in Figure S11b. The appearance of WZ/ZB mixed-phases may be due to the lattice mismatch between NW and Si (111) substrate. ${ }^{5,6}$ HRTEM images of the NW showing GaAs/GaAsSb core-shell structure and the FFT patterns in the inset demonstrate the single-crystal structure, as shown in Figure S11c-S11h. 


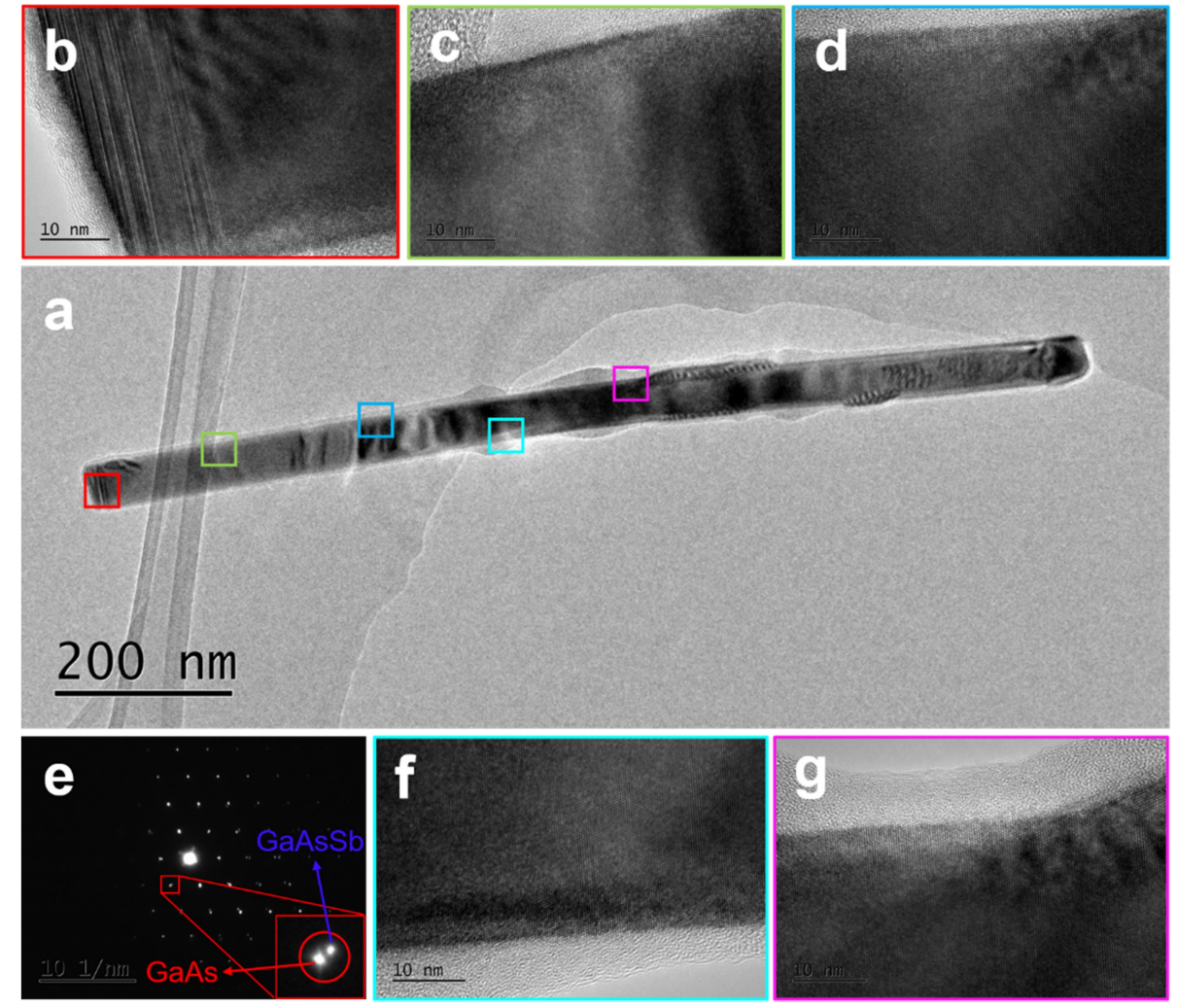

Figure S12. Structure of a typical GaAs/GaAsSb core-shell NW. (a) TEM image of another GaAs/GaAsSb core-shell NW. (b-d) and (f-g) HRTEM images taken along [111] zone axis from the bottom to middle regions of the GaAs/GaAsSb NW are shown in (a), respectively. (e) A typical SAED pattern of the selected region of the NW from panel (d). Inset shows the corresponding red box details.

Figure S12a shows that the WZ/ZB mixed-phases approximately $10 \sim 15 \mathrm{~nm}$ appears at the bottom of the GaAs/GaAsSb NW. Nevertheless, the HRTEM images show that the crystal phase of the entire NW is a pure ZB structure and free of stacking faults (Figure S12c, d, f and g). Moreover, the SAED pattern reveals that the crystalline phase is pure ZB phase and the inset shows two distinct sets of diffraction spots in the red circle, labeled as GaAs and GaAsSb, as shown in Figure S12, panel e. 


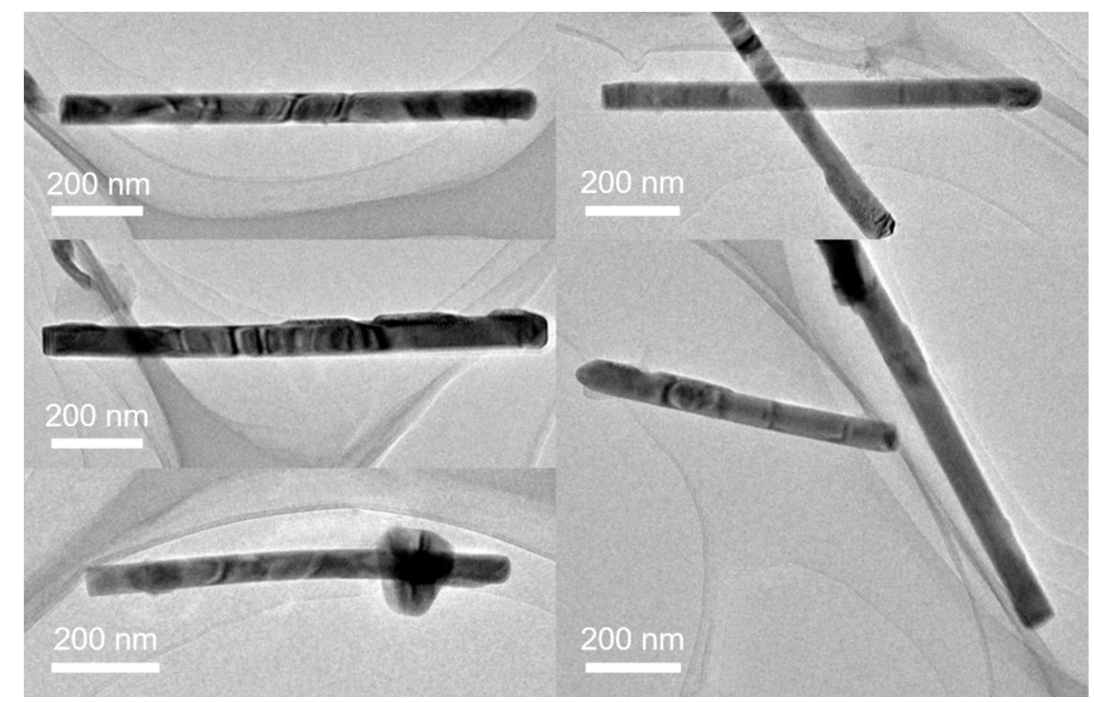

Figure S13. TEM images of GaAs/GaAsSb core-shell NWs.
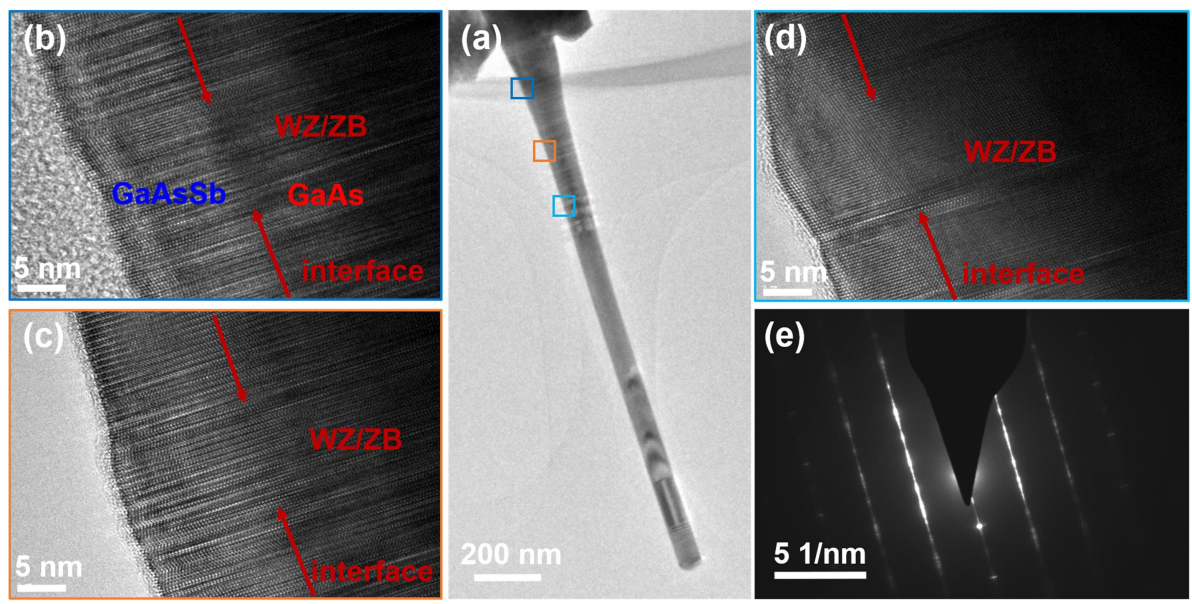

Figure S14 Structure of a typical GaAs/GaAsSb core-shell NW. (a) TEM image of another GaAs/GaAsSb core-shell NW. (b-d) HRTEM images taken along [111] zone axis from the bottom regions of the GaAs/GaAsSb core-shell NW are shown in (a), respectively. (e) A typical SAED pattern of the selected region of the NW from panel (c).

Figure S14a shows that the WZ/ZB mixed phase appears at the bottom of the GaAs/GaAsSb core-shell NW. Meanwhile, the HRTEM images and SAED pattern show that the crystal phase of the GaAs/GaAsSb core-shell NW is WZ/ZB mixed structure (Figure S14 b-e). In addition, the HRTEM images revealed that the diameter of GaAs core is $\sim 65 \mathrm{~nm}$. 

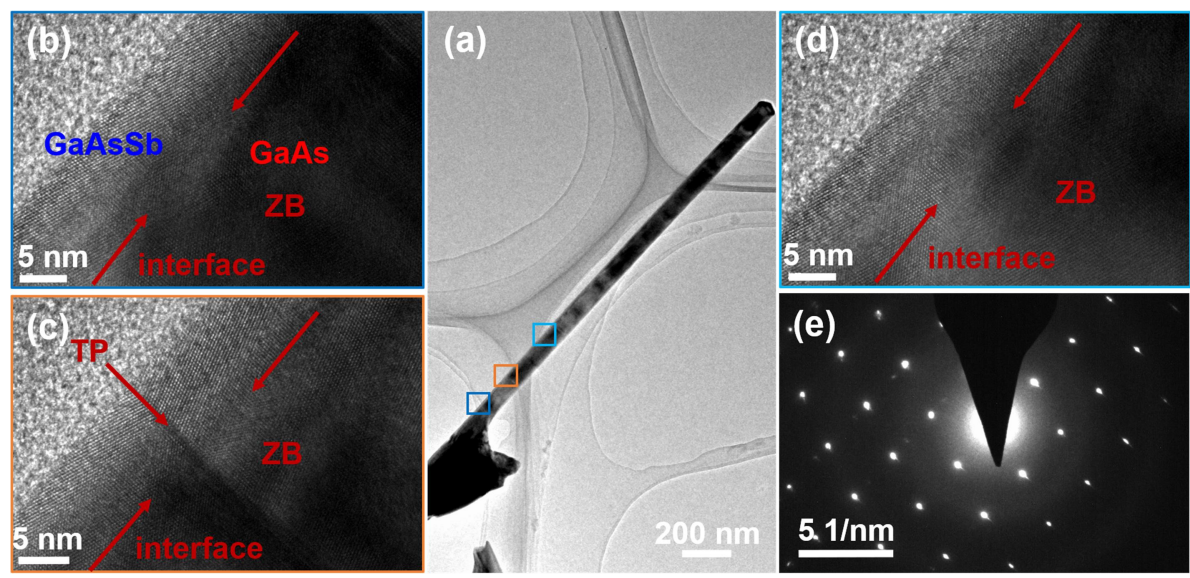

Figure S15 Structure of a typical GaAs/GaAsSb core-shell NW. (a) TEM image of another GaAs/GaAsSb core-shell NW. (b-d) HRTEM images taken along [111] zone axis from the bottom regions of the GaAs/GaAsSb core-shell NW are shown in (a), respectively. (e) A typical SAED pattern of the selected region of the NW from panel (c).

Figure S15a shows that the ZB phase appears at the bottom of the GaAs/GaAsSb core-shell NW. Meanwhile, the HRTEM images and SAED pattern show that the crystal phase of the GaAs/GaAsSb core-shell NW is ZB structure (Figure S15b, $d$ and e). Nevertheless, the HRTEM image of Figure S15c shows that the crystal phase is ZB structure with a twin plane. In addition, the HRTEM images revealed that the diameter of $\mathrm{GaAs}$ core is $\sim 60 \mathrm{~nm}$.
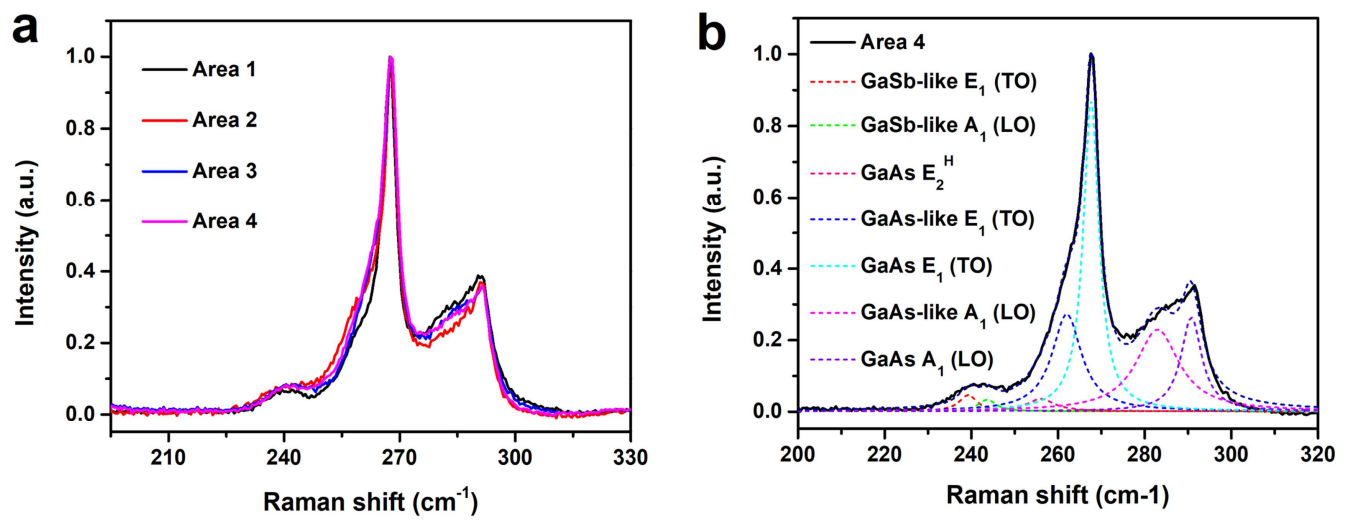

Figure S16. Raman spectra of large-area GaAs/GaAsSb NWs with a larger diameter of the GaAs core. (a) Raman spectra of GaAs/GaAsSb NWs in 4 different regions. (b) Lorentzian line fitting of GaAs/GaAsSb NWs (Area 4). 


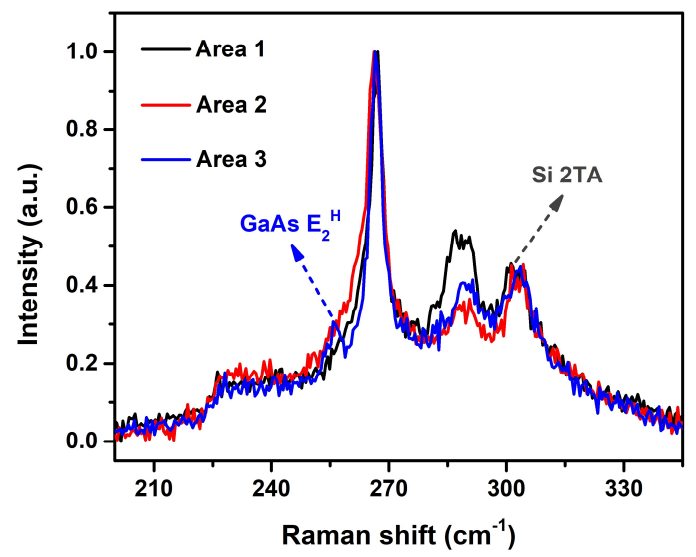

Figure S17. Raman spectra of large number dispersed GaAs/GaAsSb NWs with a larger diameter of the GaAs core.

The large-area Raman measurement of GaAs/GaAsSb NWs with a larger GaAs core has been performed and the data is shown in Figure S16. Figure S16a shows that the Raman spectra from 4 different regions of the GaAs/GaAsSb NWs, which shows similar shapes. Lorentzian fitting of the data (Area 4) is shown in Figure S16b. It can be seen clearly that the GaAs/GaAsSb NWs display the modes of GaSb-like $\mathrm{E}_{1}(\mathrm{TO})$, GaSb-like $A_{1}(\mathrm{TO})$, GaAs-like $\mathrm{E}_{1}(\mathrm{TO})$, GaAs $\mathrm{E}_{1}(\mathrm{TO})$, GaAs-like $\mathrm{A}_{1}(\mathrm{LO})$ and GaAs $\mathrm{A}_{1}(\mathrm{LO})$. Moreover, the $\mathrm{E}_{2}{ }^{\mathrm{H}}$ mode $\left(\sim 256.0 \mathrm{~cm}^{-1}\right)$ is contained in a wider Raman peak, which can only be obtained by fitting. In order to further confirm the existence of GaAs $\mathrm{E}_{2}{ }^{\mathrm{H}}$ mode peak, GaAs/GaAsSb NWs were mechanically transferred to the $\mathrm{Si}$ substrate, and their Raman measurement is shown in Figure S17. Similarly, the Raman data from 3 different regions of the GaAs/GaAsSb NWs shows similar result. In addition to the modes corresponding to the GaAs/GaAsSb NWs, the Si 2TA mode peak $\left(\sim 303.2 \mathrm{~cm}^{-1}\right)$ can be assigned to the Si substrate. ${ }^{7}$ The Raman spectrum of $\mathrm{GaAs} / \mathrm{GaAsSb} \mathrm{NWs}$ with a larger diameter of the GaAs core has obvious $\mathrm{E}_{2}{ }^{\mathrm{H}}$ mode peak $\sim 256.2 \mathrm{~cm}^{-1}$, corresponding to the WZ structure. Therefore, it can be determined 
that WZ structures exist in the GaAs/GaAsSb NWs with a large GaAs core, which is consistent with the HRTEM characterizations (Figure S14 and S15).

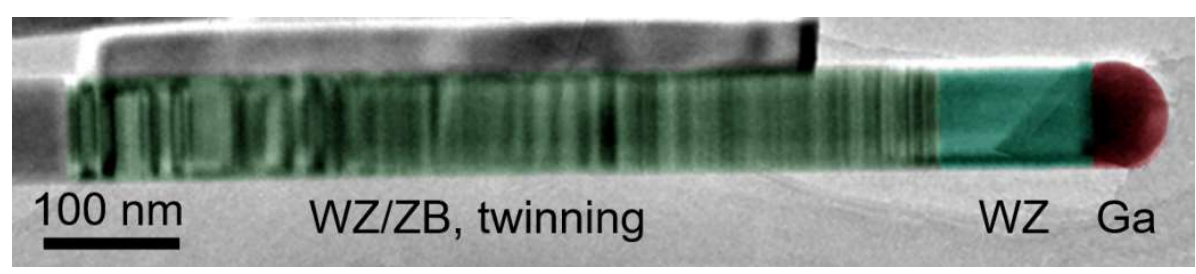

Figure S18. Structure of a typical GaAs NW. TEM image showing the crystal structure of the GaAs NW. A clear WZ/ZB mixed phase with twinning, WZ structure and Ga droplet from left to right.
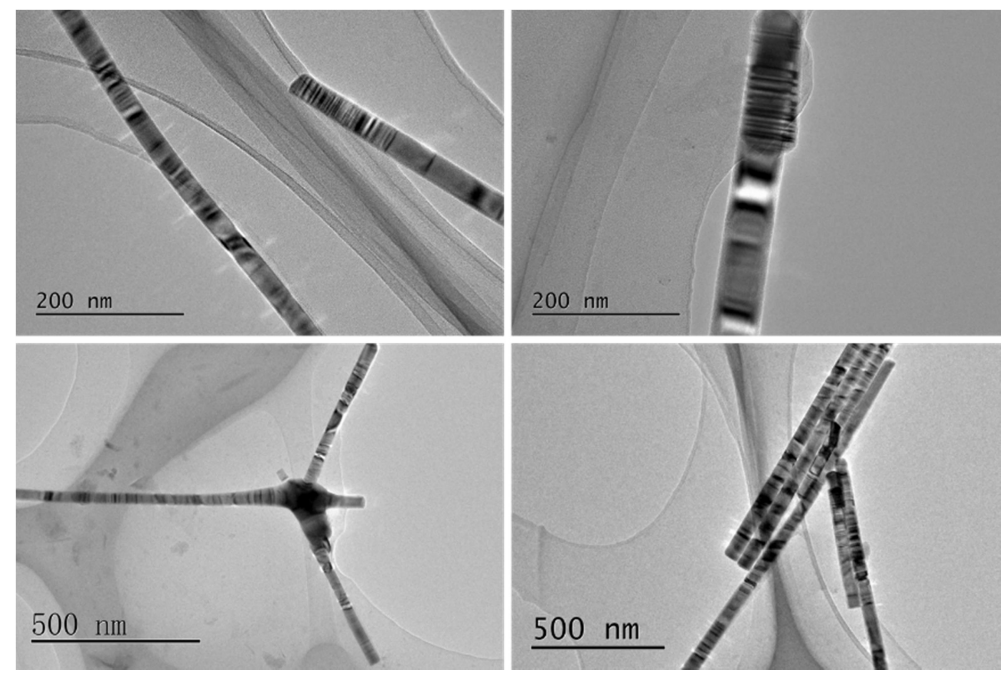

Figure S19. TEM images of GaAs NWs. The TEM of multiple GaAs NWs is shown as a mixed-phases structure.

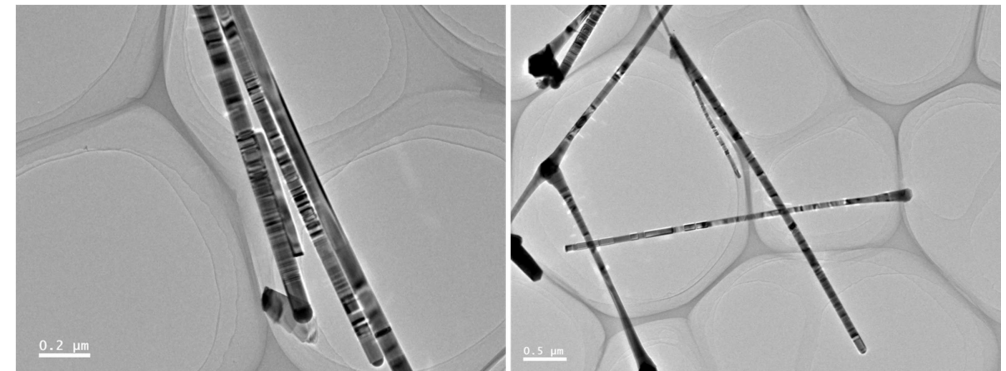

Figure S20. TEM images of GaAs NWs. The TEM of multiple GaAs NWs is shown as a mixed-phases structure. 

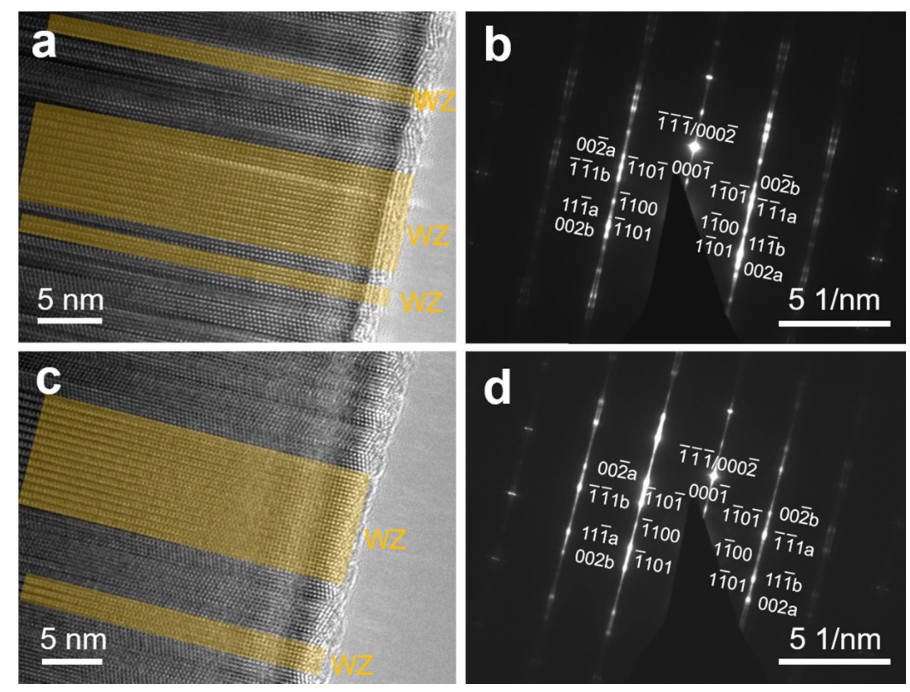

Figure S21. Structure of a typical GaAs NW. $(a, c)$ HRTEM images of two GaAs NWs taken along [111]/[0001] zone axis direction. The yellow regions are WZ structure, and the other regions are ZB structure with twin plane and stacking faults. (b,d) The SAED pattern taken for GaAs NWs confirming that both WZ and ZB mixed-phases are present.

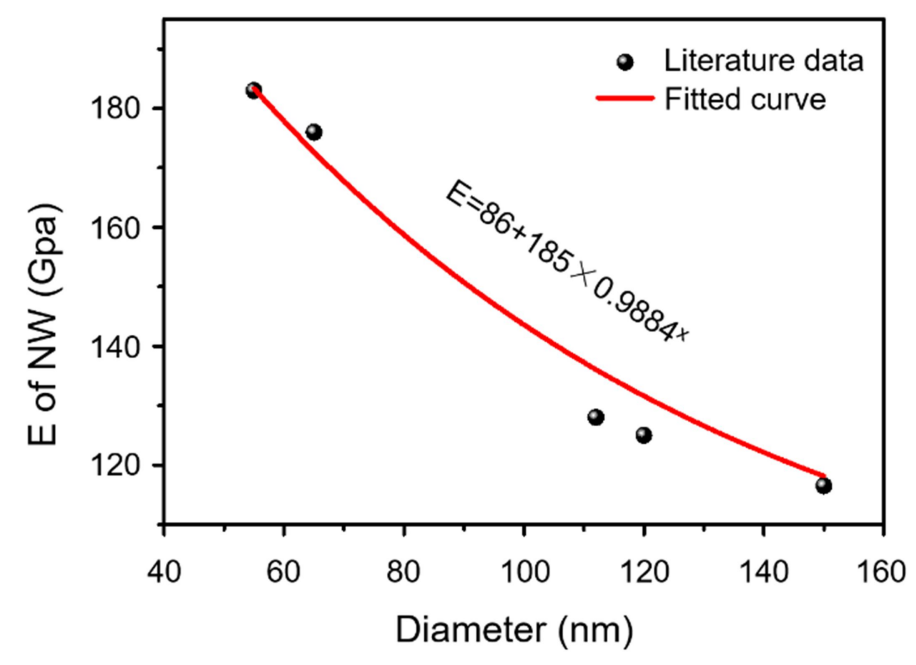

Figure S22. Young's modulus of GaAs NWs. Relationships between Young's modulus, and average diameter of GaAs NWs obtained from $\mathrm{ref}^{8,9}$ and the fitting curve obtained our GaAs NWs Young's modulus. 


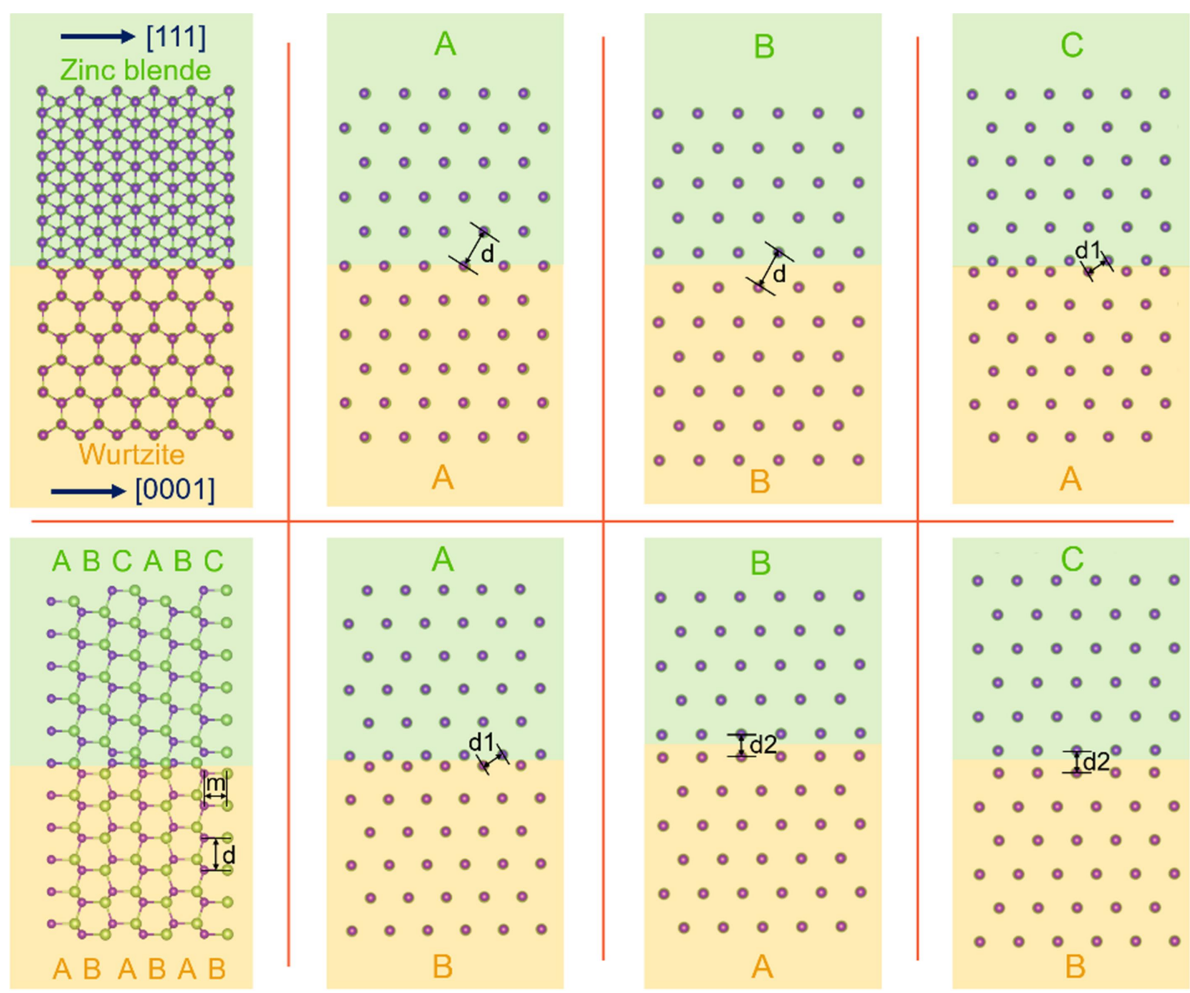

Figure S23. Transition mechanism of the transformations from WZ to ZB. Atomic structures, showing $\{0001\}$ plane stacking in WZ structure with stacking order symbolized as ...ABABAB.... and $\{111\}$ plane stacking in $\mathrm{ZB}$ structure with stacking order symbolized as ...ABCABC.... The Ga-As bond length is recorded as $\mathrm{m}$, and the distance between Ga-Ga or As-As is recorded as d.

Here, we propose a transition mechanism of the transformations from $\mathrm{WZ}$ to ZB.

Figure S23 shows the $\{0001\}$ plane stacking in a WZ structure of GaAs. Following the plane stacking in the hexagonal-close-packed (hcp) structure, the $\{0001\}$ plane stacking in the $\mathrm{WZ}$ structure can be denoted as ...ABABAB.... And Figure S23 shows the $\{111\}$ plane stacking in the ZB structure of GaAsSb. Following the $\{111\}$ plane stacking in the face-centered-cubic (fcc) structure, the $\{111\}$ plane stacking in the ZB structure can be denoted as ...ABCABC....

Under the action of stress, the reforming mechanism model in the atomic plane is shown in Figure S23. Before the atomic plane reforming, the atomic arrangement of GaAs WZ structure and GaAsSb ZB structure is carried out, and the model of WZ 
structure ...ABABAB ... to $\mathrm{ZB}$ structure ...ABCABC... is established. In the $\mathrm{C}$ and $\mathrm{A}$ layers of ZB structure, the distance between the two adjacent As atoms at the interface are denoted as $\mathrm{d} 1$, and in the $\mathrm{B}$ and $\mathrm{C}$ layers, and the distance between two adjacent As atoms are denoted as d2, as shown in Figure S23. The corresponding strain $\varepsilon$ can be given as ${ }^{10-12}$

$$
\varepsilon=\frac{L_{1}-L_{0}}{L_{0}}=\frac{\Delta L}{L_{0}}
$$

$L_{0}$ is the distance between atoms after atomic surface reforming, and $L_{1}$ is the distance between atoms before atomic surface reforming, $\Delta L$ is a deformation variable. The strain between layer $\mathrm{C}$ and layer $\mathrm{A}$ is recorded as $\varepsilon_{1}$, and the strain between layer $\mathrm{B}$ and layer $\mathrm{C}$ is recorded as $\varepsilon_{2}, \varepsilon_{1}=\varepsilon_{2}=(\sqrt{3}-1) / \sqrt{3}$. The atomic distance before and after atomic surface reforming between layer $\mathrm{C}$ and layer $\mathrm{A}$ is recorded as $\Delta a_{1}, \Delta a_{1}=\sqrt{3} d / 3$, and the atomic distance before and after atomic surface reforming between layer B and layer $\mathrm{C}$ is recorded as $\Delta a_{2}, \Delta a_{2}=\sqrt{3} d / 6$. The cell area of a single GaAs WZ structure is $S_{1}=4 d m / 3, d=2 \sqrt{6} \mathrm{~m} / 3$, $S_{1 s}=4 m \times 2 \pi R / 3$. The total potential barrier in the C-layer or A-layer atomic surface reforming is recorded as $W_{1 s}$, that is $W_{1 s}=\varepsilon 1 E S_{1 s} \Delta a_{1}$, there the $E$ is Young's Modulus of GaAs NWs. The total potential barrier in the B-layer or C-layer atomic surface reforming is recorded as $W_{2 s}$, that is $W_{2 s}=\varepsilon_{2} E S_{2 s} \Delta a_{2}$, there, $\varepsilon_{1}=\varepsilon_{2}$, $S_{1 s}=S_{2 s}, \Delta a_{2}=\Delta a_{1} / 2$. Therefore, the total potential barrier obtained by atomic plane reforming of GaAs WZ structure under stress is denoted as $W_{s}$, that is, $W_{s}=2 W_{1 s}+2 W_{2 s}$. The radius of GaAs NWs is $R$, the total cell number of GaAs WZ 
structure is denoted as $M$, then $M=\left(\pi R^{2}\right) /\left(\frac{1}{2} d \frac{\sqrt{3}}{2} d\right)$, the total number of GaAs is recorded as $M_{s}=6 M$. Consequently, due to the atomic surface reforming under stress, the WZ structure of GaAs NWs is transformed into ZB structure, and the energy is recorded as $W_{\text {GaAs }}$, that is, $W_{\text {GaAs }}=W_{s} / M_{s}$. The maximum energy of phase transition for each GaAs atom pair obtained from the interface stress of core-shell NWs with different GaAs core diameters is calculated. As the diameter of the GaAs core increases, the maximum energy of each GaAs atom pair to obtain a phase transition decreases. The theoretical calculation of the maximum energy barrier for the phase transition is $0.22 \mathrm{eV} / \mathrm{GaAs}$ atom pair, and the corresponding critical diameter of the GaAs core is $55 \mathrm{~nm}$. When the diameter of GaAs core is smaller than $55 \mathrm{~nm}$, the maximum energy of each atom pair obtained from the interface stress exceeds the critical phase transition value of $0.22 \mathrm{eV} / \mathrm{GaAs}$ atom pair. When the diameter of the GaAs core exceeds $55 \mathrm{~nm}$, the maximum energy obtained by the interfacial stress is less than the critical value for phase transition, which cannot fulfill the requirement for phase transition to occur. 


\section{REFERENCES}

(1) Kresse, G.; Furthmüller, J. Efficient iterative schemes for ab initio total-energy calculations using a plane-wave basis set. Phys. Rev. B 1996, 54, 11169-11186.

(2) Kresse, G.; Furthmüller, J. Efficiency of ab-initio total energy calculations for metals and semiconductors using a plane-wave basis set. Comput. Mater. Sci. 1996, 6, $15-50$.

(3) Perdew, J. P.; Burke, K.; Ernzerhof, M. Generalized Gradient Approximation Made Simple. Phys. Rev. Lett. 1996, 77, 3865-3868.

(4) Grimme, S. Semiempirical GGA-type density functional constructed with a long-range dispersion correction. J. Comput. Chem. 2006, 27, 1787-1799.

(5) Tomioka, K.; Kobayashi, Y.; Motohisa, J.; Hara, S.; Fukui, T. Selective-Area Growth of Vertically Aligned GaAs and GaAs/AlGaAs Core-Shell Nanowires on Si(111) Substrate. Nanotechnology 2009, 20, 145302.

(6) Tomioka, K.; Motohisa, J.; Hara, S.; Fukui, T. Control of InAs Nanowire Growth Directions on Si. Nano Lett. 2008, 8, 3475-3480.

(7) Qin, L.; Teo, K. L.; Shen, Z. X.; Peng, C. S.; Zhou, J. M. Raman scattering of Ge/Si dot superlattices under hydrostatic pressure, Phys. Rev. B 2001, 64, 075312.

(8) Wang, Y. B.; Wang, L. F.; Joyce, H. J.; Gao, Q.; Liao, X. Z.; Mai, Y. W.; Tan, H. H.; Zou, J.; Ringer, S. P.; Gao, H. J.; Jagadish, C. Super Deformability and Young's Modulus of GaAs Nanowires. Adv. Mater. 2011, 23, 1356-1360.

(9) Chen, Y. J.; Gao, Q.; Wang, Y. B.; An, X. H.; Liao, X. Z.; Mai, Y.- W.; Tan, H. H.; Zou, J.; Ringer, S. P.; Jagadish, C. Determination of Young's Modulus of Ultrathin Nanomaterials. Nano Lett. 2015, 15, 5279-5283.

(10) Lee, J. H.; Pin, M. W.; Choi, S. J.; Jo, M. H.; Shin, J. C.; Hong, S. G.; Lee, S. M.; Cho, B.; Ahn, S. J.; Song, N. W.; Yi, S. H.; Kim, Y. H. Electromechanical Properties and Spontaneous Response of the Current in InAsP Nanowires. Nano Lett. 2016, 16, 6738-6745.

(11) Dick, K. A.; Kodambaka, S.; Reuter, M. C.; Deppert, K.; Samuelson, L.; Seifert, W.; Wallenberg, L. R.; Ross, F. M. The Morphology of Axial and Branched Nanowire Heterostructures. Nano Lett. 2007, 7, 1817-1822.

(12) Yang, S.; Liu, F.; Wu, C.; Yang, S. Tuning Surface Properties of Low Dimensional Materials via Strain Engineering. Small 2016, 12, 4028-4047. 